\title{
Thalidomide Combined with Chemotherapy in Treating Patients with Advanced Colorectal Cancer
}

\author{
Xin-En Huang ${ }^{1}$, Xiao-Chun Yan ${ }^{2}$, Lin Wang ${ }^{1}$, Zhu-Qing Ji ${ }^{1}$ Li Li $^{1}$, Meng-Yan Liu ${ }^{1}$, \\ Ting Qian ${ }^{1}$, Hui-Ling Shen ${ }^{3}$, Han-Gang Gu${ }^{4}$, Yong Liu ${ }^{5}$, Ming Gu ${ }^{6}$, Li-Chun Deng ${ }^{7}$
}

\begin{abstract}
Objective: To assess the safety and effectiveness of thalidomide (produced by CHANGZHOU PHARMACEUTICAL FACTORY CO.LTD) combined with chemotherapy in treating patients with advanced colorectal cancer. Method: A consecutive cohort of pretreated patients with advanced colorectal cancer were treated with thalidomide combined with chemotherapy. And chemotherapy for patients with advanced colorectal cancer were administered according to the condition of patients. Thalidomide was orally administered at a dosage of $50 \mathrm{mg} / \mathrm{day}$ to $150 \mathrm{mg} / \mathrm{day}$ before sleeping for at least 14 days. After at least 14 days of treatment, safety and side effects were evaluated. Results: There were 12 female and 3 male patients with advanced cancer recruited into this study, including 9 patients with colon, 6 patients with rectal cancer. The median age of patients was 57(4182) years. Partial response was observed in 2 patients $(2 / 15)$, and stable disease in 3 patients(3/15). Incidences of Grade 1 to 2 myelosuppression was observed in 1/15 patients, and Grade 1 to 2 elevation of hepatic enzyme was recorded in 1/15 patients. Adverse effects on the gastrointestinal tract were documented in 1/15 patients, and were Grade 1. No Grade 3-4 toxicities were diagnosed. No treatment related death was found. Conclusions: Thalidomide combined with chemotherapy was safe and mildly effective in treating patients with advanced colorectal cancer. However, further study should be conducted to clarify the effectiveness of this combination.
\end{abstract}

Keywords: Thalidomide - chemotherapy - advanced colorectal cancer

Asian Pac J Cancer Prev, 16 (17), 7867-7869

\section{Introduction}

It was suggested by worldwide investigation that 14.1 million new cancer cases, and 8.2 million cancer-related deaths occurred, as well as 32.6 million people would live with cancer in 2012 (WHO, 2012). And among this, colorectal cancer (CRC) is one of the commonest cancers in both men and women with an estimated annual incidence of 1 million new cases (Ferlay et al., 2010), and the third most commonly diagnosed cancer and the third leading cause of cancer death worldwide (Jemal et al., 2011). Chemotherapy is an important component for patients with $\mathrm{CRC}$, especially for those with advanced or metastatic disease. However, within these decades, the standard chemotherapeutic agent for advanced or metastatic CRC is still considered to be $5-\mathrm{FU}$ or 5-FU combinations. Response rates to 5-FU alone are less than $20 \%$ and a combination regimen with biochemical modulating agents (i.e., leucovorin) seems improved response rate, while survival has not been affected. Now, multiple other agents are available for treatment of metastatic CRC. These agents include UFT, capecitabine, CPT-11, oxaliplatin and raltitrexed.
All have predictable toxicities with very similar response rates and survival. Based on phase II data, UFT gave a response rate of $30 \%$ (95\% CI: $14 \%-46 \%$ ) with a median time to progression of 7.4 months and a median survival time of 14.2 months (Nogue et al., 1996). Capecitabine had very similar response rates with 7 of 36 patients on continuous treatment, 9 of 32 on intermittent treatment and 8 of 33 patients on intermittent treatment with leucovorin. Median times to progression were 17, 30 and 24 weeks, respectively. A good safety profile was also noted (Findlay et al., 1997). How to improve the treatment efficacy and to reduce side effects are the topics of clinical studies.

Previous reorts suggested that thalidomide could be active against a variety of cancers eg., myeloma, renal cell carcinoma, Kaposi's sarcoma, and colorectal cancer (Rajkumar et al., 2000). The combination of thalidomide and irinotecan has been used in treating patients with metastatic colon cancer. Phase I-II study demonstrated that irinotecan at a dose of 300-350 mg/m2every 3 weeks and thalidomide at a dose of $400 \mathrm{mg} /$ day could be safe (Govindarajan R et al., 2002). In animal models, the concomitant treatment with irinotecan and thalidomide did not significantly alter plasma pharmacokinetics of

${ }^{I}$ Department of Chemotherapy, the Affiliated Jiangsu Cancer Hospital of Nanjing Medical University \& Jiangsu Institute of Cancer Research, Nanjing, ${ }^{2}$ Department of Medical Oncology, Huai An Cancer Hospital, Huai'An, ${ }^{3}$ Zhenjiang First People's Hospital, ${ }^{4}$ Jisngsu University Hospital, Zhenjiang, ${ }^{5} \mathrm{Xu}$ Zhou Central Hospital, Xu Zhou, ${ }^{6}$ Yan Cheng Third Hospital, Yan Cheng, ${ }^{7}$ Jiang Yin People's Hospital, JiangYin, China*For correspondence: huangxinen06@163.com 
irinotecan (Yang et al., 2005), and in the treatment of advanced or metastatic cancer, thalidomide was modest active when combined with cytotoxic agents (Fine HA et al., 2003).

On this background, we hypothesize that thalidomide combined with chemotherapy could be established as an optimal schedule in treating Chinese patients with advanced or metastatic colorectal cancer.

\section{Materials and Methods}

\section{Eligiblility criteria}

Patients recruited in this study were required to be pathologically/ cytologically diagnosed with colorectal cancer in Jiangsu Cancer Hospital \& Research Institute; to sign an informed consent before treatment; to expose to long term chemotherapy or supportive care; to have a score of karnofsky performance status (KPS) $\geq 60$ with expectancy life span more than 3 moths; to be classified with no contraindications for chemotherapy; to have a routine blood test performed 0 to 3 days before chemotherapy and normal hematopoietic function as evidenced by white blood cell count 3000/ul and platelet count 100000/ul, normal hepatic function test (aspartate aminotransaminase and alanine aminotransferase less than 1.5 times of the upper limit of normal values), renal function test (serum total bilirubin $<1.5 \mathrm{mg} / \mathrm{dl}$ and creatinine $<1.5 \mathrm{mg} / \mathrm{dl}$ ). Exclusion criteria included history of alcoholic intoxication, diabetes, and patients who were pregnant or nursing. Chemotherapy was administered according to NCCN guideline. Thalidomide was orally administered at a dosage of $50 \mathrm{mg} /$ day to $150 \mathrm{mg}$ /day before sleeping for at least 14 days. After at least 14 days of treatment, safety and side effects were evaluated.

\section{Toxicity Evaluation}

The incidence rates of toxicity in this study were assessed at baseline and respectively after two cycles of treatment, the grade of toxicities was determined according to The National Cancer Institute Common Terminology Criteria for Adverse Events (NCI CTCAE; version 3).

\section{Results}

There were 12 female and 3 male pretreatred patients with advanced cancer recruited into this study, including 9 patients with colon, 6 patients with rectal cancer. The median age of patients was 57 (41-82) years. Partial response was observed in 2 patients $(2 / 15)$, and stable disease in 3 patients (3/15). Incidences of Grade 1 to 2 myelosuppression was observed in $1 / 15$ patients, and Grade 1 to 2 elevation of hepatic enzyme was recorded in $1 / 15$ patients. Adverse effects on the gastrointestinal tract were documented in $1 / 15$ patients, and were Grade 1. No Grade 3-4 toxicities were diagnosed. No treatment related death was found.

\section{Discussion}

Thalidomide is a potent teratogen, also with anti- inflammatory and antiangiogenic activity and was newly applied in treating patients with cancer (D'Amato RJ et al., 1994). The mechanism of thalidomide in treating cancer patients involving immunomodulatory activity which is considered to inhibit the production of TNF- $\alpha$, IL-1 $\beta$, IL-6, IL-12, and granulocyte macrophage colony-stimulating factor. In primary human $\mathrm{T}$ cells, and thalidomide induces an IL-2-mediated proliferation and increases IFN- $\gamma$ production (Bartlett JB et al., 2004; Haslett PA et al., 1998). Thalidomide also inhibits $\mathrm{NF}-\varkappa \mathrm{B}$, a critical regulator of inflammatory processes (Keifer JA et al., 2001). Moreover, thalidomide inhibits IL-1 $\beta$-induced IL- 8 production, nuclear translocation of NF- $x$ B, degradation of I $x-\mathrm{B} \alpha$ (Jin SH et al., 2002), and LPS-induced COX-2 (Fujita J et al., 2001). In addition to effects on classic immune cell populations, the effect of thalidomide on tissue-specific microvascular endothelial populations, specifically the gut microvascular endothelium, during carcinogenesis and inflammatory remains a topic of research. Microvascular endothelial cells are a critical cell population in carcinogenosis, inflammation, wound healing, and inflammatory response. Angiogenesis, the growth of new microvessels, plays a key physiological role in wound healing while also playing a pathophysiological role in tumor growth, diabetic retinopathy, atherosclerosis, and chronic inflammation (Folkman J et al., 1995). Angiogenesis is regulated through a balance of positive and negative regulatory mediators. VEGF is one of the most important positive angiogenic factors (Battegay EJ et al., 1995; Veikkola T et al., 2000). VEGF plays an essential role in endothelial proliferation and angiogenesis during embryogenesis as well as the menstrual cycle, and tumogenosis (Carmeliet $\mathrm{P}$ et al., 1996; Gerber HP et al., 1999). Enhanced expression of VEGF may contribute to pathological mechanisms in adenocarcinoma, and chronic inflammation (Folkman J et al., 1995). The importance of angiogenesis in the disease processes has been demonstrated by the success of antiangiogenic therapeutic trials, which are approved for the treatment of advanced colorectal adenocarcinoma (Prat A et al., 2007). VEGF plays a key role in tumor neovascularization (Ferrara N, et al., 1999; Plate KH, et al., 1992; Shweiki D et al., 1992). And, it was hypothesized that the mechanism of anti-angiogenesis could be due to suppression of thalidomide through an induction of cell surface adhesion molecules in human umbilical vein endothelial cells (Geitz H et al., 1996).

Clinical investigation on thalidomide in treating patients with gastrointestinal cancer is limited. A previous study exploring the effects of thalidomide in refractory progressing metastatic colorectal cancer was published in 2003 (Lago LD et al., 2003). In this study, 17 patients who had failed the standard chemotherapy were entered. Patients were treated with thalidomide at a dose of 200 $\mathrm{mg}$ /day with an increased dose of $200 \mathrm{mg}$ /day every 2 weeks until a final daily dose of $800 \mathrm{mg} /$ day was achieved. One patient was removed from the study due to toxicities. There were no objective responses or stable disease. Median OS was 3.6 months. In this study, our purpose is to assess the safety and effectiveness of thalidomide (produced by CHANGZHOU PHARMACEUTICAL 
FACTORY CO.LTD) combined with chemotherapy in treating patients with advanced colorectal cancer. We recruited 12 female and 3 male patients with advanced cancer recruited into this study, including 9 patients with colon, 6 patients with rectal cancer. The median age of patients was 57 (41-82) years. All patients were treated with thalidomide combined with chemotherapy. And chemotherapy for patients with advanced colorectal cancer were administered according to the condition of patients. Thalidomide was orally administered at a dosage of $50 \mathrm{mg} /$ day to $150 \mathrm{mg}$ /day before sleeping for at least 14 days. Partial response was observed in 2 patients $(2 / 15)$, and stable disease in 3 patients (3/15). Incidences of Grade 1 to 2 myelosuppression was observed in $1 / 15$ patients, and Grade 1 to 2 elevation of hepatic enzyme was recorded in $1 / 15$ patients. Adverse effects on the gastrointestinal tract were documented in $1 / 15$ patients, and were Grade 1. No Grade 3-4 toxicities were diagnosed. No treatment related death was found. In conclusion, our current study suggested that thalidomide combined with chemotherapy was safe and mildly effective in treating patients with advanced colorectal cancer. However, further study should be conducted to clarify the effectiveness and the best combination of thalidomide with chemothereapy.

\section{Acknowledgements}

Dr. Xin-En Huang is supported by Traditional Chinese Medicine Scientifi Research Project (LZ11091) and Jiangsu Province fourth stage "333 high- level Personnel Training Project" third levels of talent cultivating object.

\section{References}

Bartlett JB, Dredge K, Dalgleish AG et al (2004). The evolution of thalidomide and its IMiD derivatives as anticancer agents. Nat Rev Cancer, 4, 314-22.

Battegay EJ (1995). Angiogenesis: mechanistic insights, neovascular diseases, and therapeutic prospects. J Mol Med, 73, 333-46.

Carmeliet P, Ferreira V, Breier G, et al (1996). Abnormal blood vessel development and lethality in embryos lacking a single VEGF allele. Nature, 380, 435-9.

D'Amato RJ, Loughnan MS, Flynn E, et al (1994). Thalidomide is an inhibitor of angiogenesis. Proc Natl Acad Sci USA, 91, 4082-5.

Ferlay J, Shin HR, Bray F, et al ( 2010). Estimates of worldwide burden of cancer in 2008: GLOBOCAN 2008. Int J Cancer, 127, 2893-917.

Ferrara N (1999). Molecular and biological properties of vascular endothelial growth factor. $J$ Mol Med, 77, 527-43.

Findlay M, Van Cutsem E, KochaW, et al (1997). A randomized phase II study of capecitabine in patients with advanced colorectal cancer. Proc Am Soc Clin Oncol, 16, 227a (Abstr).

Fine HA, Wen PY, Maher EA, et al (2003). Phase II trial of thalidomide and carmustine for patients with recurrent highgrade gliomas. J Clin Oncol, 21, 2299-304.

Folkman J (1995). Angiogenesis in cancer, vascular, rheumatoid and other disease. Nat Med, 1, 27-31.

Folkman J (1995). Seminars in Medicine of the Beth Israel Hospital, Boston. Clinical applications of research on angiogenesis. N Engl J Med, 333, 1757-63.

Fujita J, Mestre JR, Zeldis JB, et al (2001). Thalidomide and its analogues inhibit lipopolysaccharide-mediated induction of cyclooxygenase-2. Clin Cancer Res, 7, 3349-55.

Geitz H, Handt S, Zwingenberger K (1996). Thalidomide selectively modulates the density of cell surface molecules involved in the adhesion cascade. Immunopharmacology, 31, 213-21.

Gerber HP, Hillan KJ, Ryan AM, et al (1999). VEGF is required for growth and survival in neonatal mice. Development, 126, 1149-59.

Govindarajan R (2002). Irinotecan/thalidomide in metastatic colorectal cancer. Oncology (Huntingt), 16, 23-6.

Haslett PA, Corral LG, Albert M, et al (1998). . Thalidomide costimulates primary human $\mathrm{T}$ lymphocytes, preferentially inducing proliferation, cytokine production, and cytotoxic responses in the CD8+ subset. J Exp Med, 187, 1885-1892.

Jemal A, Bray F, CenterMMet al (2011). Global cancer statistics. CA Cancer J Clin, 61, 69-90.

Jin SH, Kim TI, Han DS, et al (2002). Thalidomide suppresses the interleukin 1beta-induced NFkappaB signaling pathway in colon cancer cells. Ann NY Acad Sci, 973, 414-8.

Keifer JA, Guttridge DC, Ashburner BP, et al (2001). Inhibition of NF-kappa B activity by thalidomide through suppression of IkappaB kinase activity. J Biol Chem, 276, 22382-7.

Lago LD, Richter MF, Cancela AI, et al ( 2003). Phase II trial and pharmacokinetic study of thalidomide in patients with metastatic colorectal cancer. Invest New Drugs, 21, 359-66.

Nogue M, Segui MA, Batiste E et al (1996). Phase II study of oral Tegafur (TF) and low-dose oral leucovorin in advanced colorectal cancer. Proc Am Soc Clin Oncol, 15, 200 (Abstr).

Plate KH, Breier G, Weich HA, et al (1992). Vascular endothelial growth factor is a potential tumour angiogenesis factor in human gliomas in vivo. Nature, 359, 845-8.

Prat A, Casado E, Cortes J (2007). New approaches in angiogenic targeting for colorectal cancer. World J Gastroenterol, 13, 5857-66.

Rajkumar SV, Witzig TE (2000). A review of angiogenesis and antiangiogenic therapy with thalidomide in multiple myeloma. Cancer Treat Rev, 26, 351-62.

Shweiki D, Itin A, Soffer D, et al (1992 ). Vascular endothelial growth factor induced by hypoxia may mediate hypoxiainitiated angiogenesis. Nature, 359, 843-5.

Veikkola T, Karkkainen M, Claesson-Welsh L, et al (2000). Regulation of angiogenesis via vascular endothelial growth factor receptors. Cancer Res, 60, 203-12,

Yang X, Hu Z, Chan SY, et al (2005). Simultaneous determination of the lactone and carboxylate forms of irinotecan (CPT-11) and its active metabolite $\mathrm{SN}-38$ by high-performance liquid chromatography: application to plasma pharmacokinetic studies in the rat. J Chromatogr B Anal Technol Biomed Life Sci, 821, 221-8. 